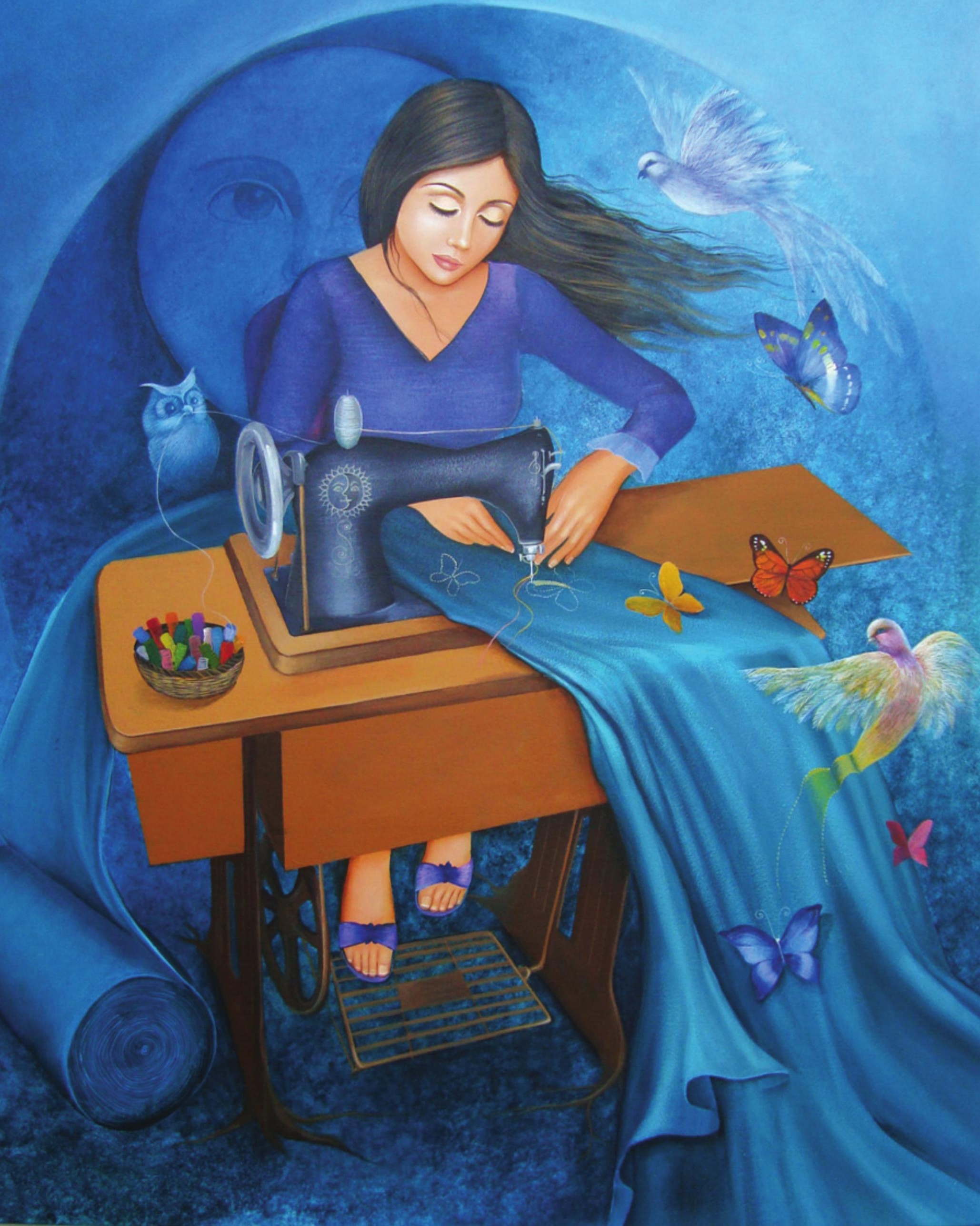




\section{Empleabilidad y la Inserción Ocupacional de la Fuerza de Trabajo en los/as Jóvenes de 12 a 30 Años en Honduras, 2006 y 2010} Héctor Alcides FigueroA*

RESUMEN. La principal razón por la cual corresponde ocuparse del mercado de trabajo y determinar los factores económicos sociodemográficos que condicionan la empleabilidad y la inserción ocupacional de la fuerza de trabajo en los/as jóvenes de 12 a 30 años en Honduras, es por la estrecha relación que tiene el empleo con el bienestar de las familias o para ponerlo en negativo, con la evolución de la pobreza, pues la gente es pobre por que no tiene empleo o el empleo que posee no le proporciona ingresos suficientes. Los/as jóvenes comprenden un segmento importante de la población en Honduras, este grupo poblacional enfrenta problemas de empleabilidad situación que se ve agravada por los bajos niveles educativos que no les permite desarrollar las competencias que exige el mercado laboral. En general, la preparación que reciben los/as jóvenes de Honduras y la capacidad demandada en el mercado laboral muestra un desfase pues la calidad y cobertura de la educación son insuficientes para satisfacer las exigencias en la demanda en los mercados de trabajo o para mantener o mejorar la empleabilidad de la Población Económicamente Activa (PEA).

Palabras clave: jóvenes, empleabilidad, inserción ocupacional, fuerza de trabajo, población económicamente activa, población en edad de trabajar.

ABSTRACT. The principal reason for take care of the labor market, is determine the economical social-demography facts who determine the employability and the occupational insertion of the workforce in the youth between 12 and 30 years in Honduras, is for the close relationship that has the employment with the family health or putting in a negative way with the poverty evolution, so the people is poor because they do not have works, or the work they have not provides them sufficient income. The youth have an important segment in Honduras population, this group faces problems of employability and this situation seems aggraded for the low educational levels that do not provides them develop the competitions that labor market demands. In general, the preparation that the youth in Honduras receive and the capacity demand in labor market shows a mismatch because the education quality and coverage are insufficient to satisfy the demand evolution in the labors markets or to hold or improve the employability of Economical Active Population (EAP).

Keywords: youth, employability, occupational insertion, work force, EAP (Economical Active Population) population with age for work.

\section{Introducción}

El presente estudio se orienta al conocimiento y análisis de los factores económicos y sociodemográficos que condicionan la empleabilidad y la inserción ocupacional de la fuerza de trabajo en los/as jóvenes de 12 a 30 años en Honduras en los años 2006 y 2010.

En el contexto nacional el estudio pretende identificar la fuerza laboral atendiendo sus principales características o variables: Población en Edad de Trabajar(PET), Población Económicamente Activa (PEA), tasa de par- ticipación, tasa de desempleo abierto, subempleo invisible y subempleo visible; personas ocupadas que declaran ingresos, ingresos promedio de las personas ocupadas, experiencia laboral y nivel educativo.

La investigación ofrece información y datos que puedan servir para sustentar los procesos de diseño e instrumentos de políticas, planes, programas o proyectos de desarrollo económico y social de instituciones públicas o privadas.

La empleabilidad remite a la capacidad de las personas para aprovechar las oportunidades de educación

Recibido: abril 2012/Aceptado y versión final agosto 2012

* Máster en Demografía Social UNAH, hectorencon@yahoo.es 
y de formación que se presenten con miras a encontrar y conservar un trabajo decente, progresar en la empresa o cambiar de empleo y adaptarse a la evolución de la tecnología y de las condiciones del mercado de trabajo ${ }^{1}$.

En general, la preparación que reciben los/as jóvenes de Honduras y la capacidad demandada en el mercado laboral muestra el desfase que posee la fuerza de trabajo, pues el contenido, la calidad y la cobertura de la educación son insuficientes para satisfacer las exigencias de la demanda en los mercados de trabajo o para mantener o mejorar la empleabilidad de la PEA.

\section{Metodología}

La investigación se desarrolló con un enfoque explicativo, estadístico y demográfico, se estudiaron dos periodos 2006 y 2010.

La investigación se enfoca en los factores sociodemográficos que condicionan la empleabilidad y la inserción ocupacional de la fuerza de trabajo joven de Honduras. El estudio se orientó a identificar los factores, características y tendencias que presenta el mercado laboral y la participación de los/as jóvenes en la inserción; para ello se seleccionaron 4 variables de estudio: sexo, nivel educativo, categoría ocupacional y rama de actividad.

La unidad de análisis son los/as jóvenes de 12 a 30 años de edad desagregados en 4 intervalos.

El procesamiento de datos se realizó por medio del uso de paquetes estadísticos como SPSS que es el formato en el que se elaboran las bases de datos de las Encuetas Permanentes de Hogares de Propósitos Múltiples (EPHPM). Para la elaboración de cuadros y gráficos se utilizó la herramienta de Microsoft Excel.

\section{La Población Joven de 12 a 30 Años en Hon- duras (2006-2010).}

Este capítulo presenta datos generales de la población joven en Honduras relacionados con distribución según área de residencia, nivel de escolaridad, población joven en el mercado laboral y $\mathrm{PET}^{2}$ según área de residencia y nivel educativo.

\subsection{Población Joven Según Área de Residencia}

Para el 2006 el 52\% de la población joven residía en el área rural y un $48 \%$ en las zonas urbanas. Del total de esta población $52 \%$ eran mujeres y $48 \%$ hombres. La población joven predominantemente en el área urbana era la femenina.

La distribución por sexo en la zona urbana, fue de $54 \%$ para las mujeres y $46 \%$ para los hombres, mientras que en la zona rural la población masculina y la femenina son similares (Gráfico No.1). Quizá este fenómeno esté relacionado con la migración de las mujeres del campo a la ciudad donde se insertan al mercado laboral generalmente en las maquilas y los servicios.

En el 2010, la población joven presenta similares características donde $47 \%$ reside en las zonas urbanas y $53 \%$ en las zonas rurales, la población joven de hombres y mujeres muestra una distribución uniforme, se denota la diferencia cuando se desagrega por zona de residencia, en la zona urbana $52 \%$ eran mujeres y $48 \%$ hombres, mientras que la zona rural el comportamiento es a la inversa $52 \%$ eran hombres y $48 \%$ mujeres (Gráfico No.1).

1. Empleabilidad, recomendación 195 de la OIT, 2004.

2. Población en Edad de Trabajar: población de 10 años y más. 
Gráfico No. 1. Honduras: Población Joven por Sexo, Según Zona de Residencia, 2006-2010

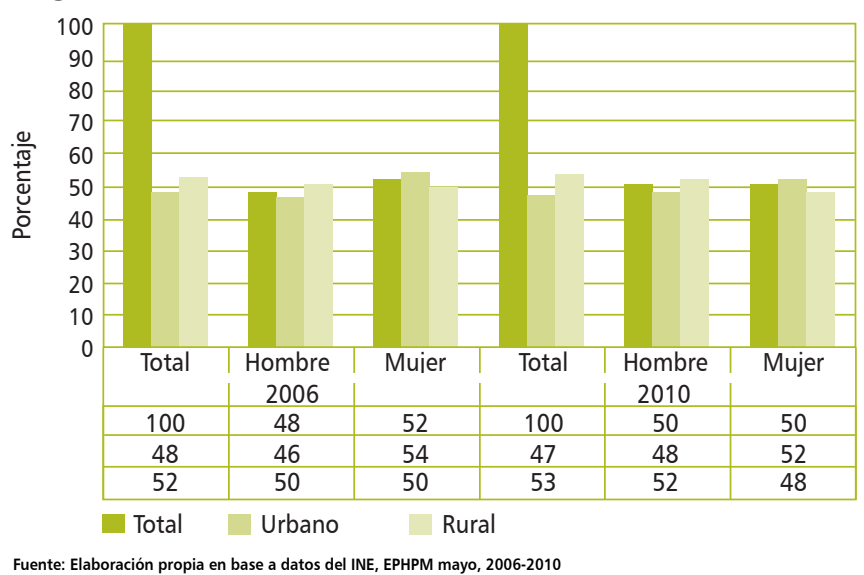

3.2 Población Joven de 12 a 30 Años por Sexo Según Rango de Edad

Atendiendo a la distribución por sexo se observa que en el 2006, los/as jóvenes que se encontraban en el rango de 12 a 14 años representaban 43\%; de los cuales 23\% eran hombres y $21 \%$ mujeres; población bastante similar en cuanto a su distribución, las diferencias más marcadas se observan en los rangos de edad de 19 a 24 años, en donde el $30 \%$ son mujeres y $28 \%$ hombres.

En el 2010, se mantiene la tendencia y la proporción mayor de la población joven se concentra en el rango de edad de 19 a 24 años, donde 30\% eran hombres y 31\% mujeres (Gráfico No.2).

Gráfico No. 2. Honduras: Población Joven por Sexo, Según Rango de Edad, 2006-2010

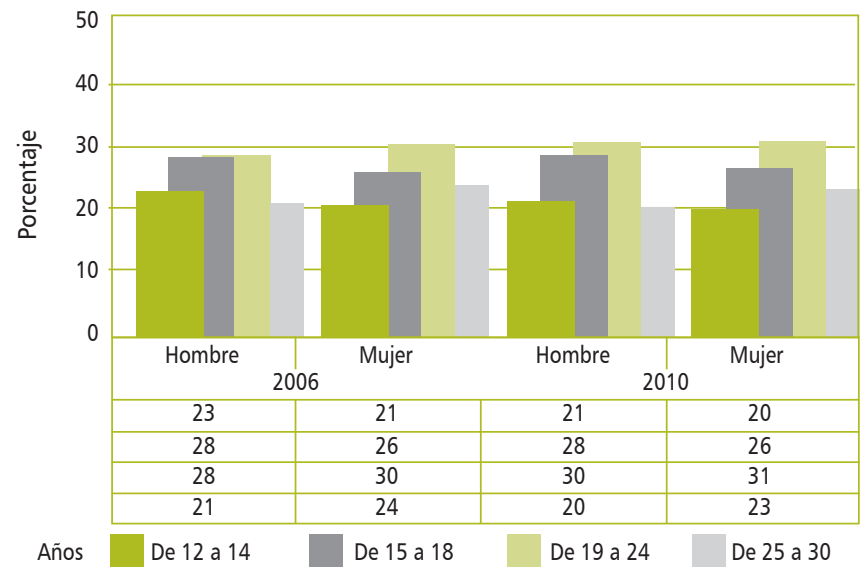

Fuente: Elaboración propia en base a datos del INE, EPHPM mayo, 2006-2010
Los primeros dos rangos, es decir, los que se encuentran entre 12 y 18 años, se caracterizan por su grado de dependencia económica y presentan mayor vulnerabilidad por encontrarse en el ciclo de vida de la adolescencia, etapa que se vive con mayor intensidad y las decisiones que se toman determinarán en gran medida su destino y su desempeño en el mercado laboral.

La Población en Edad de Trabajar en Honduras en el 2006, representaba 38\% de la población total, y en el 2010, esta población representó 39\%, aumentó 1 punto porcentual.

3.3 Población en Edad de Trabajar (PET) Según Área de Residencia y Nivel Educativo

La PET en Honduras se concentra en el área rural, en el 2006, 52\% vivían en estas zonas y en el 2010 $53 \%$, esto debido a que las familias en las zonas rurales son más numerosas que en las zonas urbanas (Gráfico No.3). El tamaño de los hogares en el 2006, era de 5.1 en promedio; en el 2010 bajó a 4.7 .

Teniendo en cuenta el nivel educativo en el 2006, $6 \%$ de la PET, no contaba con ningún nivel educativo, $52 \%$ contaba con nivel primario, $35 \%$ con secundaria, y un $6 \%$ con nivel superior.

En el 2010, 4\% de la PET no contaba con ningún nivel educativo, $48 \%$ contaba con educación primaria,

Gráfico No. 3. Población en Edad de Trabajar, Según Área de Residencia, Nivel Educativo, 2006-2010

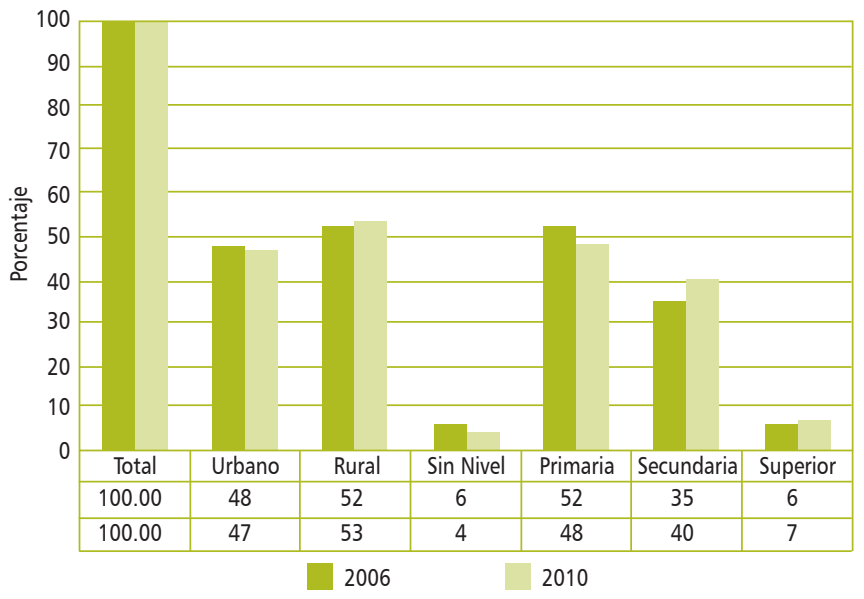

Fuente: Elaboración propia en base a datos del INE, EPHPM mayo, 2006-2010 
$40 \%$ con secundaria y $7 \%$ con nivel superior. En el 2010, la población que no tenía ningún nivel educativo se redujo en $1.9 \%$ puntos porcentuales con respecto al 2006 , el nivel superior de la PET aumentó en 1.1 con respecto al 2006 (Gráfico No.3).

Es importante destacar la importancia de la educación en la población joven y sobre todo la cobertura y la calidad, ya que si los/as jóvenes se educan tendrán mejores oportunidades en el mercado laboral. A nivel nacional, en relación a los años de estudio promedio ${ }^{3}$ de la PET en el 2006 era de 7 años; (8 años en el área urbana, 6 años en la rural). En el 2010, no se muestra una mejoría en la educación de los/as jóvenes, se mantienen 7 años de estudio, donde se presenta una diferencia es al desagregarlos por área de residencia ( 9 años en el área urbana y 6 en el área rural). (Cuadro No.1).

\section{Nivel de Pobreza de la Población Joven de}

\section{2-30 Años e Ingresos Económicos}

La información de la Encuesta Permanente de Hogares de Propósitos Múltiples, permite establecer relaciones entre sus variables e inferir ciertas asociaciones entre la pobreza y las características personales de los miembros del hogar.

Es importante relacionar el desempleo y la pobreza, el problema del desempleo juvenil es más grave entre los sectores más pobres esto se debe a varios factores, entre ellos: sus bajos niveles de escolaridad y calificación, la falta de redes y servicios de apoyo.

En el 2006, 58\% de los/as jóvenes de Honduras estaban en condición de pobreza. El 2010 muestra una reducción de 1 punto porcentual respecto al 2006 (Cuadro No.2).

Cuadro No. 1. Honduras: Población Joven de 12 a 30 Años Según Dominio, Nivel Educativo y Rango de Edad por Sexo, 2006-2010

\begin{tabular}{|c|c|c|c|c|c|c|}
\hline \multirow{2}{*}{ Categorías } & \multicolumn{3}{|c|}{2006} & \multicolumn{3}{|c|}{2010} \\
\hline & Total & Hombre & Mujer & Total & Hombre & Mujer \\
\hline Total & 100 & 48 & 52 & 100 & 50 & 50 \\
\hline \multicolumn{7}{|l|}{ Domino } \\
\hline Urbano & 48 & 46 & 50 & 47 & 45 & 49 \\
\hline Rural & 52 & 54 & 50 & 53 & 55 & 51 \\
\hline Nivel Educativo & & & & & 0 & 0 \\
\hline Sin Nivel & 6 & 7 & 5 & 4 & 5 & 4 \\
\hline Primaria & 52 & 55 & 50 & 48 & 52 & 45 \\
\hline Secundaria & 35 & 32 & 38 & 40 & 36 & 44 \\
\hline Superior & 6 & 6 & 7 & 7 & 7 & 8 \\
\hline $\mathrm{Ns} / \mathrm{Nr}$ & 0.1 & 0 & 0 & 0.1 & 0 & 0 \\
\hline Rango de edad & & & & & 0 & 0 \\
\hline De 12 a 14 años & 22 & 23 & 21 & 20 & 21 & 20 \\
\hline De 15 a 18 años & 27 & 28 & 26 & 27 & 28 & 26 \\
\hline De 19 a 24 años & 29 & 28 & 30 & 30 & 30 & 31 \\
\hline De 25 a 30 años & 22 & 21 & 24 & 22 & 20 & 23 \\
\hline
\end{tabular}

Fuente: Elaboración propia en base a datos del INE, EPHPM mayo, 2006-2010 
Cuadro No. 2. Honduras: Nivel de Pobreza de la Población Joven de 12-30 Años, Según Zona de Residencia, Sexo, Nivel Educativo y Rango de Edad, 2006-2010

\begin{tabular}{|c|c|c|c|c|c|c|c|c|c|c|}
\hline \multirow{2}{*}{ Categorías } & \multicolumn{5}{|c|}{2006} & \multicolumn{5}{|c|}{2010} \\
\hline & Total & No pobres & Pobres & Extrema & Relativa & Total & No pobres & Pobres & Extrema & Relativa \\
\hline Total & 100 & 42 & 58 & 36 & 22 & 100 & 43 & 57 & 35 & 22 \\
\hline Urbano & 54 & 48 & 52 & 20 & 32 & 50 & 47 & 53 & 20 & 33 \\
\hline Rural & 46 & 36 & 64 & 54 & 10 & 50 & 38 & 62 & 50 & 11 \\
\hline \multicolumn{11}{|l|}{ Sexo } \\
\hline Hombre & 83 & 43 & 57 & 35 & 22 & 77 & 43 & 57 & 36 & 21 \\
\hline Mujer & 17 & 38 & 62 & 40 & 22 & 23 & 40 & 60 & 35 & 25 \\
\hline \multicolumn{11}{|l|}{ Nivel educativo } \\
\hline Sin Nivel & 10 & 19 & 81 & 66 & 15 & 7 & 15 & 85 & 68 & 17 \\
\hline Primaria & 60 & 36 & 64 & 42 & 22 & 56 & 31 & 69 & 47 & 22 \\
\hline Secundaria & 24 & 59 & 41 & 14 & 27 & 29 & 59 & 41 & 14 & 27 \\
\hline Superior & 6 & 81 & 19 & 7 & 12 & 7 & 89 & 11 & 1 & 9 \\
\hline $\mathrm{Ns} / \mathrm{Nr}$ & 0 & 47 & 53 & 34 & 19 & 0 & 73 & 27 & 14 & 14 \\
\hline \multicolumn{11}{|l|}{ Rango de edad } \\
\hline 12 a 14 años & 0 & 0 & 0 & 0 & 0 & 0 & 0 & 0 & 0 & 0 \\
\hline 15 a 18 años & 2 & 47 & 53 & 35 & 18 & 3 & 55 & 45 & 33 & 12 \\
\hline 19 a 24 años & 33 & 46 & 54 & 30 & 25 & 33 & 47 & 53 & 31 & 22 \\
\hline 25 a 30 años & 65 & 41 & 59 & 39 & 21 & 63 & 40 & 60 & 38 & 23 \\
\hline
\end{tabular}

Fuente: Elaboración propia en base a la EPHPM mayo, 2006-2010.

En resumen, contar con una ocupación en condiciones adecuadas es la base principal para mejorar la situación de vida de la población. Sólo puede alcanzarse bienestar para los/as jóvenes y sus hogares por medio de la creación de un número suficiente de buenas ocupaciones. Por el contrario, la escasez de tales oportunidades es precursora de la pobreza y frustración de oportunidades de desarrollo personal entre la juventud.

El nivel de instrucción es determinante en el nivel de ingresos percibos por remuneración al trabajo por los/as jóvenes, por lo que una mejor educación ayuda a mejorar sus condiciones socioeconómicas.

El ingreso promedio mensual de los jóvenes ocupados que no tienen educación formal es inferior comparado con el de aquellos jóvenes que tiene educación primaria secundaria o superior.

Se denota el mayor nivel de ingreso entre más educada estén los/as jóvenes; comportamiento que se mantiene, en el 2010, indistintamente de la categoría ocupacional, si trabaja en el sector privado o público, o sólo se hace por cuenta propia (Gráfico No.4).
En el 2006, la diferencia de los ingresos entre los/as jóvenes con educación superior versus los/as jóvenes que no tienen ningún nivel de instrucción es de casi 5 veces lo que gana un joven sin nivel educativo, tendencia que se mantiene en el 2010 (Gráfico No.4).

Gráfico No. 4. Honduras: Ingreso de los/as Jóvenes, Según Nivel Educativo, 2006-2010

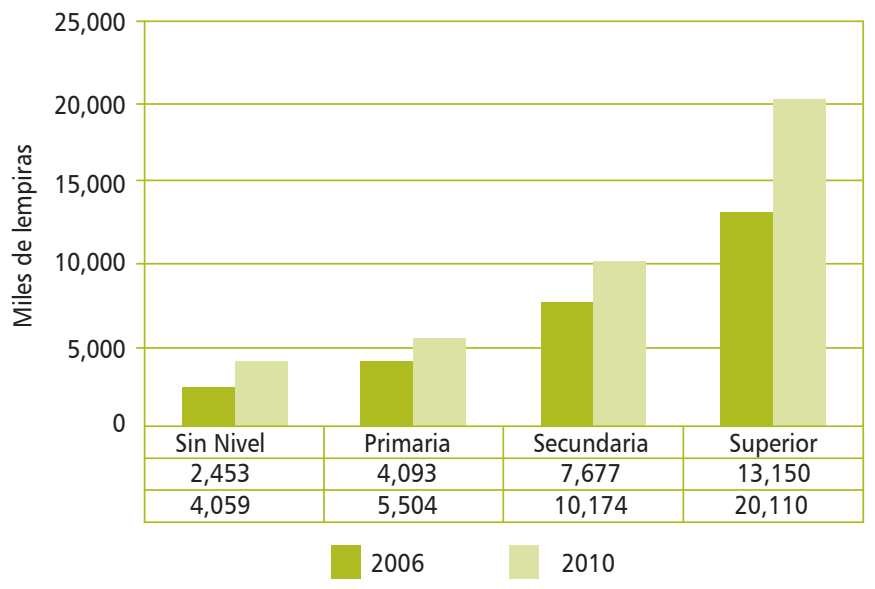

Fuente: Elaboración propia en base a la EPHPM mayo, 2006-2010. 


\section{Tendencias del Mercado Laboral}

\subsection{Población Joven Ocupada}

La evolución de los ocupados expresa un comportamiento creciente, cuyos datos reflejan una mayor participación en el mercado laboral juvenil, en este apartado se presenta la población ocupada según área de residencia, nivel educativo y rango de edad.

En el 2006 la mayor parte de la población juvenil ocupada tenía un bajo nivel educativo, 58.3\% (714,773) de los/as jóvenes sólo contaba con educación primaria y en el 2010, 54\% $(774,914)$ contaba con educación primaria denotando así el problema acentuado del mercado laboral (Cuadro No.3).

Los comentarios más importantes que suscita este cuadro se refieren en primer lugar al importante crecimiento que ha tenido la PEA nacional en los años 2006 y 2010. Y al crecimiento del desempleo y subempleo, además denota como el nivel educativo de la PEA sigue siendo bajo.

\subsection{Jóvenes en el Mercado Laboral Según Categoría Ocupacional}

$\mathrm{Al}$ analizar el mercado de trabajo juvenil se toma en cuenta el grado de heterogeneidad establecida por el peso que tenga el empleo asalariado en el mercado laboral.

En el 2006, el empleo asalariado absorbió el 57.5\% de la fuerza laboral juvenil, en el 2010, este porcentaje se reduce a $53,8 \%$, es decir $3.7 \%$. Se produce un cambio en relación a la categoría el autoempleo o los cuenta propia que absorbió en el 2006, el 21.5\% y en el 2010, 22.1\% esta relación denota una disminución en el empleo asalariado y un aumento del autoempleo y el trabajo familiar no remunerado (Gráfico No.5).

Gráfico No. 5. Honduras: Jóvenes Según Categoría Ocupacional, 2006-2010

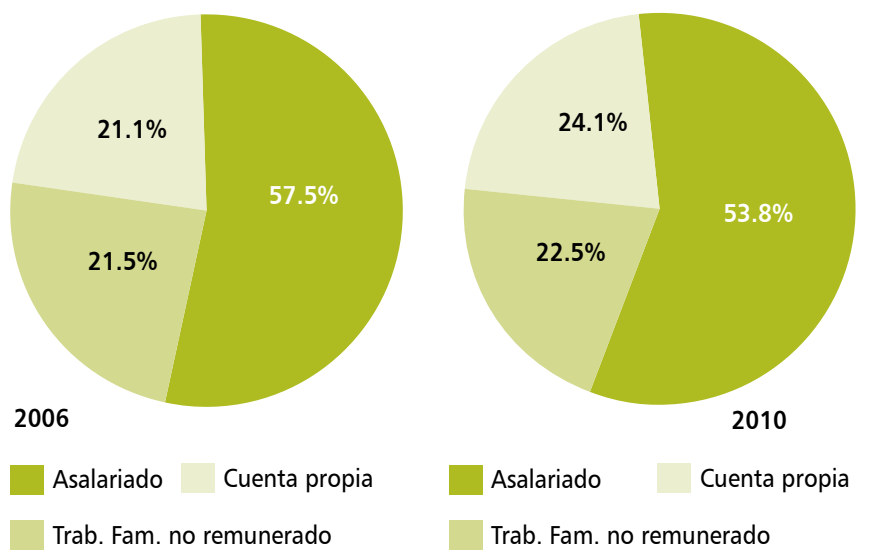

Nota: Porcentaje por fila

Fuente: Elaboración propia en base a datos del INE, EPHPM mayo, 2006-2010

Cuadro No. 3. Honduras: Población Económicamente Activa (Ocupados, Desocupados), 2006-2010

\begin{tabular}{|c|c|c|c|c|c|c|}
\hline \multirow{2}{*}{ Categorías } & \multicolumn{3}{|c|}{2006} & \multicolumn{3}{|c|}{2010} \\
\hline & PEA & Ocupados & Desocupados & PEA & Ocupados & Desocupados \\
\hline Total & 1292,615 & 1225,268 & 67,347 & 1522,723 & 1427,165 & 95,557 \\
\hline \multicolumn{7}{|l|}{ Domino } \\
\hline Urbano & 618,327 & 570,228 & 48,099 & 687,518 & 614,142 & 73,376 \\
\hline Rural & 674,288 & 655,040 & 19,248 & 835,204 & 813,023 & 22,181 \\
\hline \multicolumn{7}{|l|}{ Nivel educativo } \\
\hline Sin Nivel & 101,291 & 99,160 & 2,131 & 75,258 & 73,955 & 1,304 \\
\hline Primaria & 741,919 & 714,773 & 27,146 & 804,654 & 774,914 & 29,740 \\
\hline Secundaria & 353,650 & 323,231 & 30,419 & 514,949 & 465,339 & 49,610 \\
\hline Superior & 93,421 & 85,980 & 7,441 & 125,180 & 110,277 & 14,903 \\
\hline $\mathrm{Ns} / \mathrm{Nr}$ & 2,334 & 2,124 & 210 & 2,682 & 2,682 & 0 \\
\hline \multicolumn{7}{|l|}{ Rango de edad } \\
\hline 12 a 14 años & 91,496 & 90,979 & 517 & 115,322 & 114,412 & 910 \\
\hline 15 a 18 años & 279,698 & 267,381 & 12,317 & 332,644 & 317,380 & 15,264 \\
\hline 19 a 24 años & 497,763 & 461,705 & 36,059 & 594,188 & 540,268 & 53,920 \\
\hline 25 a 30 años & 423,657 & 405,203 & 18,455 & 480,568 & 455,106 & 25,463 \\
\hline
\end{tabular}

Fuente: Elaboración propia en base a datos del INE, EPHPM mayo, 2006-2010 
Los/as jóvenes que desarrollan actividades como cuenta propia en el 2006, eran 252,182 y en el 2010, se registraron 315,152, manifestándose en mayor proporción en el área rural en el 2006, 63.5\% y en el 2010, con $66.2 \%$ en su mayoría realizaron trabajo de subsistencia (Gráfico No.6).

Históricamente los mercados de trabajo en América Latina han sido heterogéneos, por su parte Honduras mantiene el mismo comportamiento, donde el empleo

Gráfica No. 6. Honduras: Jóvenes por Categoría Ocupacional, Según Zona de Residencia, 2006-2010

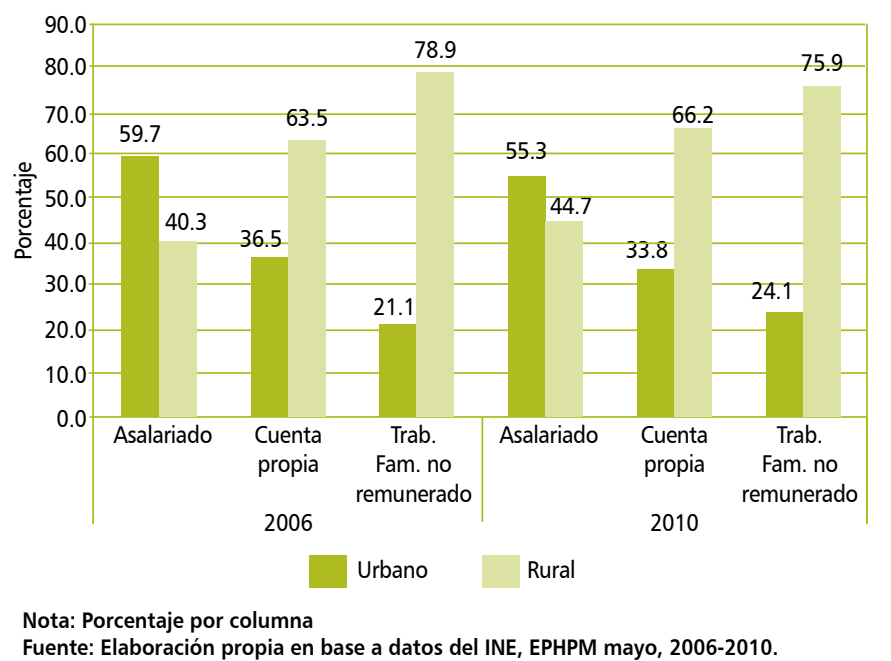

asalariado y el de cuenta propia absorben a la fuerza laboral joven.

\subsection{Jóvenes Ocupados por Rama de Actividad Económi-} ca, 2006-2010

Los sectores de agricultura, comercio y manufactura siguen siendo los grandes empleadores en el 2006, este sector absorbió $73.7 \%$ del total de los/as jóvenes ocupados; en el 2010, absorbió 74.7\%, haciendo denotar que predomina el empleo rural al encontrarse que el sector de la agricultura en el 2006 empleo al 37.1\% y el 2010 el $39.0 \%$ de los/as jóvenes, se puede observar que se mantiene la tendencia en el 2010 (Gráfico No.7).

El mercado de trabajo hondureño concentra la mayor parte de la fuerza de trabajo en la agricultura, mayoritariamente por su actividad económica tradicional, con una productividad muy baja, con algún grado de modernidad en ciertas ramas. Le sigue un sector moderno urbano y un amplio sector informal urbano y rural, denotado por subempleo y el autoempleo.

La mano de obra rural permite al sector moderno rural especializarse en cultivos de mano de obra intensiva, de bajo nivel tecnológico, cuya competitividad internacional queda garantizada por el bajo costo de los recursos

Gráfico No. 7. Honduras: Jóvenes Ocupados por Rama de Actividad Económica, 2006-2010 (En miles de personas)

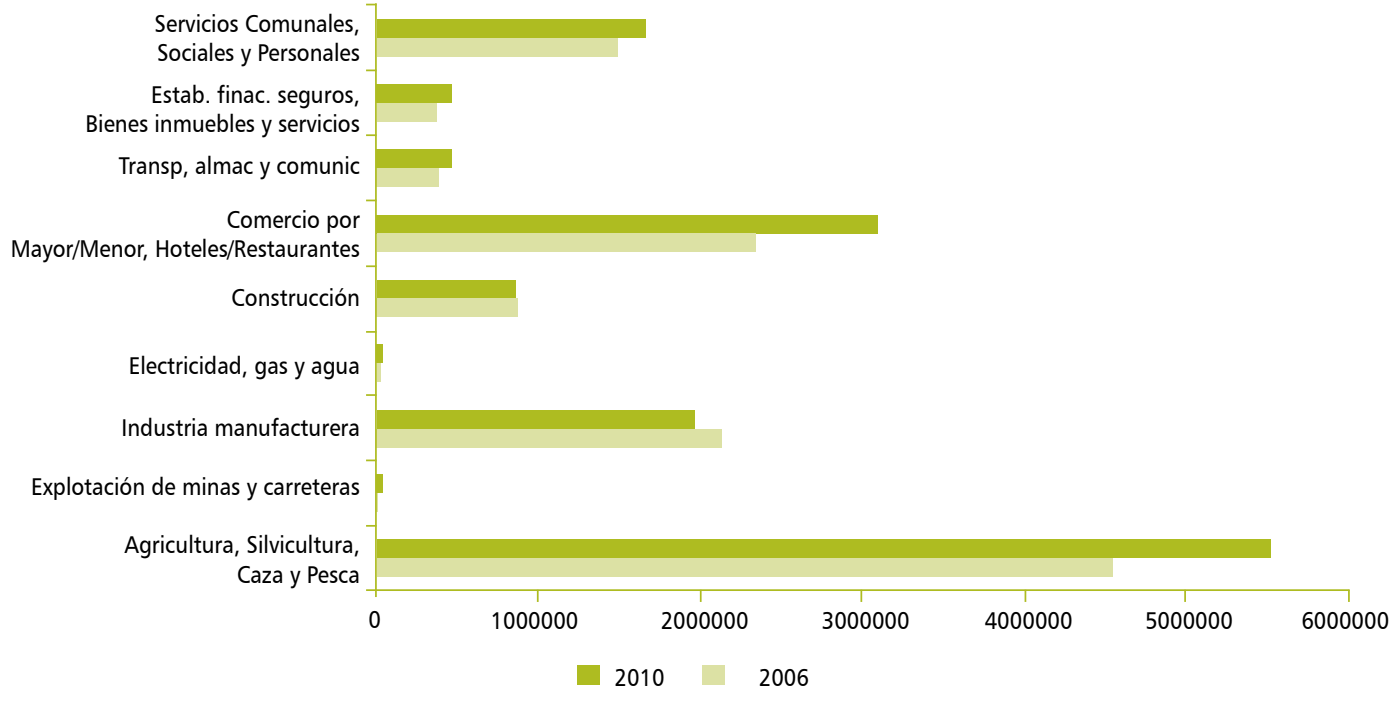

Fuente: Elaboración propia en base a datos del INE, EPHPM mayo, 2006-2010 
naturales y de la mano de obra, enfrentando entonces una oferta de recurso humano infinitamente elástica, la naturaleza ilimitada, como la descrita por el premio nóbel de economía Sir Arthur Lewis en su modelo de economía dual.

Bajo tales condiciones, el mercado de trabajo rural, presenta una situación en la cual las variaciones (los desplazamientos hacia afuera) de la curva de demanda sólo afectan el nivel de empleo, mientras las remuneraciones al trabajo permanecen al nivel de subsistencia.

El problema principal del país es la baja productividad de la fuerza laboral juvenil ${ }^{4}$, así como al desempeño de otras variables que influyen en ella como ser la inversión. Esto refleja una proporción muy alta del subempleo.

5.4 Jóvenes Frente al Mercado Laboral y la Educación, 2006-2010

La empleabilidad de los/as jóvenes y el papel que juega la educación es de suma importancia al momento de insertarse en el mercado de trabajo, es necesario preguntarse si la educación formal es suficiente para dotar de las habilidades necesarias que se requieren en el mercado laboral, si el sistema de educación formal transmite las actitudes y las condiciones para que los/as jóvenes puedan buscar, obtener y quedarse en el empleo. No cabe la menor duda que la educación es un factor estratégico para mejorar las condiciones en que los/as jóvenes pueden tener mejores empleos y mayores niveles salariales.

El Gráfico No.8, refleja la curva descendente de los/as jóvenes que sólo estudian mostrando la misma tendencia tanto en el 2006 como en el 2010, lo cual sería un comportamiento que podría decirse normal, si no iniciara a muy temprana edad, sin embargo, se observa que a partir de los 15 años inicia una caida vertiginosa lo que permite deducir una incorporación temprana al mercado laboral y deserción escolar alta.

La población que no estudia ni trabaja forma una curva ascendente tendencia que se mantiene en el 2010, a lo largo de los diferente rangos de edades hasta llegar a los 25 años, entre las edades de 12 a 14 años donde el $12.7 \%$ en el 2006 se encontraba en esta situación y en el 2010, el 11.9\%, mostrando un máximo de 31.6\% entre las edades de 25 y 30 años en el 2006, en el 2010, el máximo se da entre las edades de 19 a 24 años con $30.0 \%$. Esta situación debe ser analizada en detalle por

\section{Gráfico No. 8. Honduras: Jóvenes Frente Al Mercado Laboral y la Educación, 2006-2010}
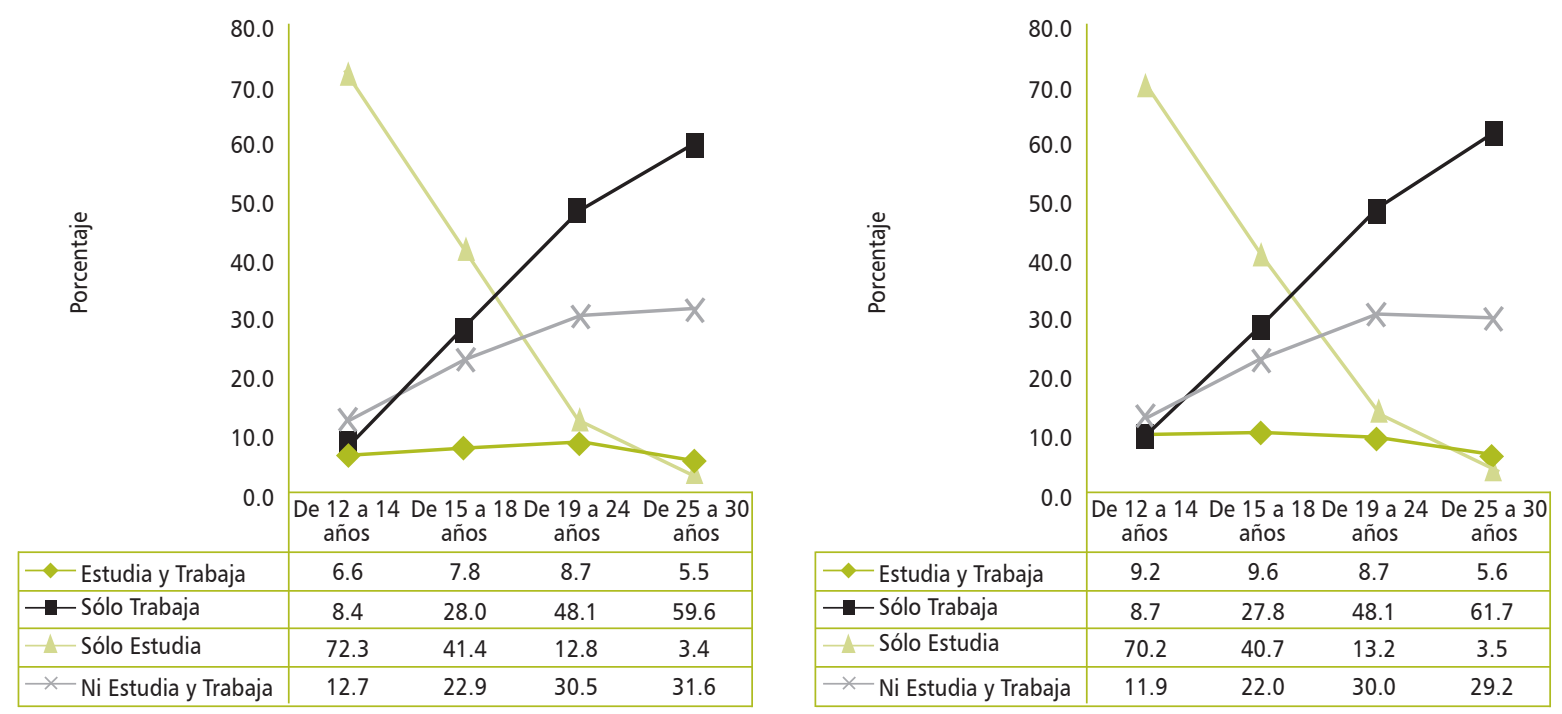

Fuente: Elaboración propia en base a datos del INE, EPHPM mayo, 2006-2010

4. Productividad laboral se refiere al valor agregado por hora trabajada 
todas las implicaciones negativas que conlleva para la sociedad hondureña (Gráfico No.8).

La curva que refleja a la población que estudia y trabaja, denota la falta de oportunidades de efectuar trabajos de medio tiempo, por temporadas o por tareas que permitan dicha combinación.

Para concluir esta sección, se tiene que insistir que desde el punto de vista de política pública, es crucial prestar especial atención a un subgrupo de aquellos/as jóvenes que solamente trabajan, de manera específica los involucrados en actividades informales y en puestos con condiciones precarias, quienes ven comprometidas sus posibilidades de acceder a una mejor educación.

5.5 Jóvenes que se Insertan por Primera Vez al Mercado Laboral.

En los países en vías de desarrollo como Honduras, con una elevada proporción de población juvenil rural y consecuentemente altos niveles de trabajadores insertos en actividades tradicionales rurales e informales urbanas, en el Cuadro No.4 se puede observar que las ramas donde se insertan los/as jóvenes por primera vez al mercado laboral. En el 2006 lo hacen mayormente en la rama de actividad primaria $33.3 \%$, en la secundaria $21.2 \%$, y en la terciaria 54,5\%. En el 2010, esta tendencia se mantiene, en la rama actividad primaria se inserta el $34.5 \%$, en la secundaria el $16.5 \%$, y en la terciaria el $49.0 \%$.

Cuando se analiza según nivel educativo al sumar a los/as jóvenes que no tienen ningún nivel educativo y los que cuenta con primaria estos representan $86.7 \%$ de los/as jóvenes que se insertan a la rama de actividad primaria, es decir gran porcentaje de ellos, trabajan en actividades en las cuales desarrollan largas jornadas, es evidente que a mayor nivel educativo de los/as jóvenes mejores serán las oportunidades de insertarse en trabajos mejor remunerados (Cuadro No.4).

Cuadro No. 4. Honduras: Jóvenes que Insertan al Mercado de Trabajo por Primera Vez, Según Nivel Educativo y Rama de Actividad, 2006-2010

\begin{tabular}{|c|c|c|c|c|c|c|c|c|c|c|}
\hline \multirow[b]{2}{*}{ Categorías } & \multicolumn{5}{|c|}{2006} & \multicolumn{5}{|c|}{2010} \\
\hline & Total & Sin Nivel & Primaria & $\begin{array}{l}\text { Secunda- } \\
\text { ria }\end{array}$ & Superior & Total & Sin Nivel & Primaria & $\begin{array}{l}\text { Secunda- } \\
\text { ria }\end{array}$ & Superior \\
\hline Total & 307,515 & 16,003 & 162,571 & 102,607 & 26,335 & 330,890 & 61,793 & 194,513 & 54,341 & 20,243 \\
\hline \multicolumn{11}{|l|}{ Dominio } \\
\hline Urbano & 151,399 & 3,568 & 53,043 & 71,435 & 23,354 & 149,017 & 14,293 & 72,666 & 43,425 & 18,634 \\
\hline Rural & 156,116 & 12,435 & 109,528 & 31,172 & 2,981 & 181,873 & 47,500 & 121,848 & 10,916 & 1,609 \\
\hline \multicolumn{11}{|c|}{ Rama de Actividad Económica } \\
\hline $\begin{array}{l}\text { Agricultura, Silvicultura, Caza } \\
\text { y Pesca }\end{array}$ & 102,458 & 10,583 & 78,280 & 12,905 & 690 & 114,297 & 35,344 & 76,816 & 1,869 & 268 \\
\hline $\begin{array}{l}\text { Explotación de minas y } \\
\text { canteras }\end{array}$ & 601 & 68 & 533 & - & - & 444 & 268 & 176 & - & - \\
\hline Industria manufacturera & 51,186 & 1,339 & 24,013 & 22,382 & 3,453 & 43,585 & 8,003 & 25,301 & 8,940 & 1,341 \\
\hline Electricidad, gas y agua & 770 & - & 200 & 367 & 204 & 1,003 & 268 & 734 & - & - \\
\hline Construcción & 13,302 & 362 & 8,440 & 3,839 & 661 & 10,527 & 1,295 & 6,687 & 1,768 & 778 \\
\hline $\begin{array}{l}\text { Comercio por Mayor/Menor, } \\
\text { Hoteles/Restaurantes }\end{array}$ & 75,223 & 1,808 & 31,224 & 35,344 & 6,846 & 92,386 & 9,505 & 54,726 & 24,211 & 3,945 \\
\hline Transp almac y comunic & 6,919 & 170 & 2,366 & 3,257 & 1,126 & 7,113 & 268 & 3,431 & 3,136 & 277 \\
\hline $\begin{array}{l}\text { Estab. finac. seguros, Bienes } \\
\text { nmuebles y servicios }\end{array}$ & 9,631 & 55 & 679 & 4,763 & 4,134 & 13,306 & 1,811 & 4,465 & 3,110 & 3,921 \\
\hline $\begin{array}{l}\text { Servicios Comunales, Sociales } \\
\text { y Personales }\end{array}$ & 47,235 & 1,550 & 16,768 & 19,696 & 9,220 & 44,478 & 3,883 & 20,205 & 10,676 & 9,713 \\
\hline
\end{tabular}

Fuente: Elaboración propia en base a datos del INE, EPHPM mayo, 2006-2010 
Es importante que el país desarrolle proyectos para los/as jóvenes del área rural que han alcanzado un nivel de educación superior ya que estos muestran un avance importante en cuanto a la formación educativa, hay que aprovechar esta mano de obra calificada.

\subsection{Ingresos Promedios y el Nivel Educativo de los/as} Jóvenes.

En general, la empleabilidad de los/as jóvenes puede ser favorecida con mejores niveles de educación. El nivel de instrucción es determinante en el nivel de ingresos percibos por remuneración al trabajo de los/as jóvenes, una mejor educación ayuda a mejorar las condiciones socioeconómicas de los/as jóvenes.

Indistintamente de la educación el ingreso promedio mensual es más alto entre los/as jóvenes con mayor educación que entre aquellos que no han tenido educación formal, tanto en el sector público o privado o si lo hacen por cuenta propia, esta tendencia se oberva tanto en el 2006 como el 2010 (Gráfico No.9).

La diferencia de ingresos entre los/as jóvenes con educación superior versus los/as jóvenes que no tienen ningún nivel de instrucción es de casi 5 veces en el año 2006, tendencia que se mantiene en el 2010 (Gráfico No.9).

Gráfico No. 9. Honduras: Ingreso de los/as Jóvenes, Según Nivel Educativo, 2006-2010

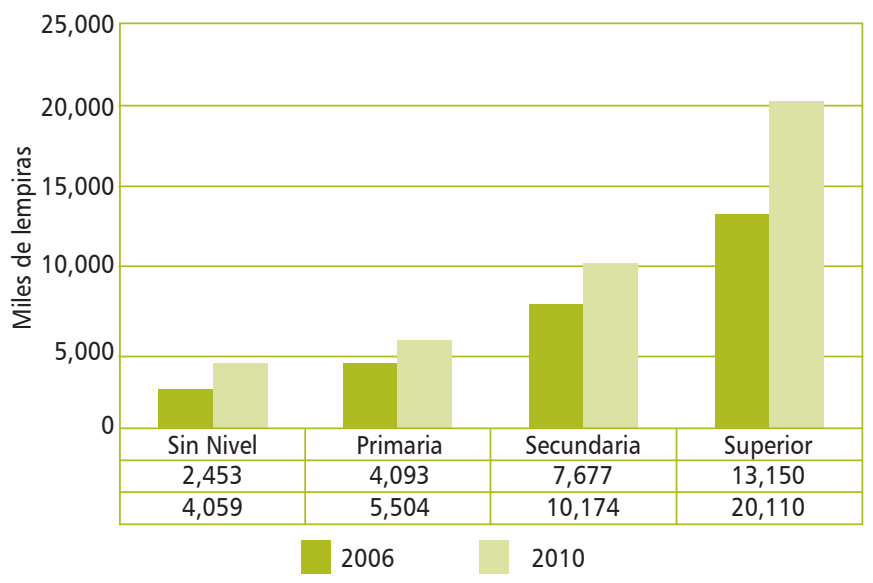

Fuente: Elaboración propia en base a la EPHPM mayo, 2006-2010.

\section{Reflexión Final}

La población joven de Honduras, representa una porción mayoritaria y sustancial de la población total del país, lo que revela el potencial de fuerza de trabajo con que cuenta la nación.

La educación juega un papel muy importante en el tema de empleabilidad juvenil, sin embargo, la oferta educativa del país, no presenta alternativas acordes a la demanda del mercado laboral, además no está diseñado para que los/as jóvenes puedan combinar estudio y trabajo lo cual es una debilidad, que obliga a los/as jóvenes en condición de pobreza a abandonar sus estudios y a incorporarse al mercado laboral.

La propuesta es que la mejora de las condiciones de empleabilidad de los/las jóvenes hondureños/as es aquí y ahora, pasa por abordar esencialmente aspectos centrales como: mejorar la oferta educativa básica y técnica tanto para los jóvenes que buscan empleo por primera vez como para los insertados en el mercado de trabajo pero en condiciones no tan satisfactorias; y favorecer la creación de empleo a través del fomento de la empresarialidad y el emprendedurismo juvenil mediante la promoción a nivel macro de una política económica que integre lo social con lo económico y que en esa medida favorezca efectivamente la creación de empleo. Además, que tenga como principio rector la forja de una alianza público-privada, que premie la productividad empresarial, la responsabilidad social y ambiental y la intensidad de mano de obra cuando esto sea posible a favor de los jóvenes.

\section{Bibliografía}

- INE (2006) Encuesta Permanente de Hogares de Propósitos Múltiples, Honduras.

- INE (2010) Encuesta Permanente de Hogares de Propósitos Múltiples, Honduras. 